\title{
Spinristor: A Swiss Army Knife of Molecular Electronics
}

Esmaeil Farajpour Bonab, ${ }^{1,2}$ Adam Jaroš,${ }^{3,4}$ Zahra Badri, ${ }^{5}$ Lucie Tučková, ${ }^{3}$ Michal Straka, $* 3$ Cina Foroutan-Nejad*3,5

${ }^{1}$ Department of Chemistry, Faculty of Science, Masaryk University, Kamenice 5, Brno, Czech Republic

${ }^{2}$ CEITEC - Central European Institute of Technology, Masaryk University, Kamenice 5/C4, CZ-62500 Brno, Czech Republic

${ }^{3}$ Institute of Organic Chemistry and Biochemistry of the Czech Academy of Sciences, Flemingovo nám. 2, CZ-16610, Prague, Czech Republic

${ }^{4}$ Faculty of Science, Charles University, Albertov 2038/6, Prague 2, 128 43, Czech Republic

${ }^{5}$ Institute of Organic Chemistry, Polish Academy of Sciences, Kasprzaka 44/52, 01-224, Warsaw, Poland

Corresponding authors' emails:

MS: straka@uochb.cas.cz

CFN: cforoutan-nejad@icho.edu.pl 


\begin{abstract}
Here, we propose and provide in silico proof of concept of a spinristor; a new molecular electronic component that combines a spin-filter, a rectifier, and a switch, in a single molecule for in-memory processing. It builds on the idea of an open-shell transition metal ion enclosed within an elliptical fullerene connected to the source, drain, and a pair of gate electrodes. The spin- and electronic polarization due to the enclosed metal leads to differential rectification of the electrons at low voltages applied between the source-drain electrodes, $\mathrm{V}_{\mathrm{SD}}$. The position of the encapsulated ion can be switched by a combination of a high $\mathrm{V}_{\mathrm{SD}}$ and a voltage applied between gate electrodes, $V_{G}$, to switch the direction of the rectification and spin-filtering ratio. The system can thus be used as a switching rectifier and spin-filter, a spinristor. To the best of our knowledge, such a system has no macroscopic counterpart in electronics.
\end{abstract}




\section{What is a spinristor?}

The holy grail of molecular electronics ${ }^{1-6}$ is the miniaturization of electronic circuits through replacing circuit components with single molecules with similar functions. After the insightful lecture of Richard Feynman, "There is plenty of room at the bottom", 7 Aviram and Ratner introduced the first examples of molecular devices, molecular diodes. ${ }^{8}$ In subsequent years, various molecular devices starting from simple wires, ${ }^{9}$ rectifiers, ${ }^{8,10}$ and switches ${ }^{11,12}$ to more complicated transistors ${ }^{13}$ and memristors ${ }^{14-19}$ have been proposed, fabricated, and some even commercialized. ${ }^{20}$

So far the general trend in molecular electronics has been replicating macroscopic circuit components on a nanoscale level. However, molecular electronics can do better. Here, we use in silico multiscale modeling to devise the first example of a molecular circuit component, that so far has no equivalent in the macroscopic world; we coin it spinristor. The spinristor is a circuit component that combines the functions of a memristor ${ }^{21,22}$ and a spinfilter. Alternatively, considering the memristor as a combination of a resistor/switch and a rectifier as proposed by Abraham, ${ }^{23}$ the spinristor can be defined as a circuit component combining the functionality of a switch, a rectifier, and a spin-filter.

\section{Design of molecular spinristors}

To design a functional spinristor, one may employ known molecular memristors and tune them to sustain spin-filtering properties. Thus far known molecular devices with memristive properties can be classified under three main groups: (1) organic molecules which undergo bond breaking/forming processes, ${ }^{12,17,24}$ (2) transition metal complexes (TMCs) whose conductivity changes upon spin-crossover or redox processes, ${ }^{14,18,19}$ and (3) endohedral fullerenes. ${ }^{15,16}$ While the relevant organic systems have a closed-shell electronic structure that rules out spin filtering properties, the TMCs can, hypothetically, enable spin-filtering properties, if the transition metals in their ground electronic state have an open-shell electronic 
structure. However, to the best of our knowledge, the spin-filtering properties for experimentally studied TMC memristors have neither been reported experimentally nor theoretically. The endohedral metallofullerenes seem to offer the functionalities needed for molecular spinristors. Our idea of a molecular spinristor consists of a transition metal ion enclosed in a fullerene cage, here $\mathrm{C}_{70}-D_{5 h}(1)$, that is connected to four electrodes, Fig. 1. The primary function of the proposed spinristor is spin-filtering when a low potential between source and drain electrodes, $\mathrm{V}_{\mathrm{SD}}$, is applied, Figs. 1ab. The spin-filtering arises from the spinpolarized electronic structure of the $\mathrm{M} @ \mathrm{C}_{70}$, vide infra. When the $\mathrm{V}_{\mathrm{SD}}$ and the gate voltage, $\mathrm{V}_{\mathrm{G}}$, increase up to a certain threshold, the enclosed metal atom relocates to a different position Fig. 1c and 1d, in an electric-field-driven switching process. This leads to a change in the overall conductivity of the system akin to a memristor, ${ }^{15}$ but, in the spinristor, also the spinfiltering ratio changes. Overall, the proposed system will function as a switching spin-filter or a spin-filtering memristor, shortly spinristor.

The so-far experimentally reported EMF switching molecular components typically remain functional at temperatures near absolute zero because their switching barriers are rather low. ${ }^{16,25,26}$ This stems mainly from the quasi-isotropic nature of the fullerene interior and largely ionic (i.e. non-directional) character of the metal-cage bonding. ${ }^{27,28}$ For example, the Gd@ $\mathrm{C}_{82}$ molecular electret operates with a barrier of ca $0.25 \mathrm{kcal} / \mathrm{mol}(11 \mathrm{meV})$ at $1.6 \mathrm{~K},{ }^{16}$ and Li@ $\mathrm{C}_{60}$, a multistate molecular switch in STM experiments, works at $5 \mathrm{~K} .{ }^{29}$ Should the switch work in realistic data storage and processing, it should ideally have an energy barrier equal to or higher than $50 \mathrm{kT}$, which turns to a comparatively large switching barrier of about $35-38 \mathrm{kcal} . \mathrm{mol}^{-1}$ at the working temperature of the present-day computers. ${ }^{30,31}$ If the energy barrier halves, the year-long stability reaches the day-long stability. ${ }^{31}$ 


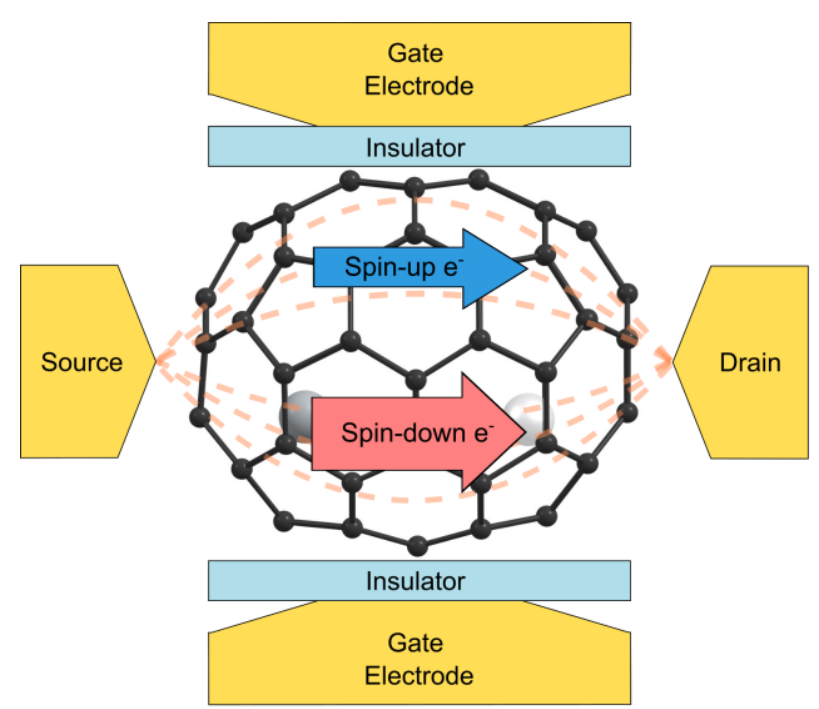

a

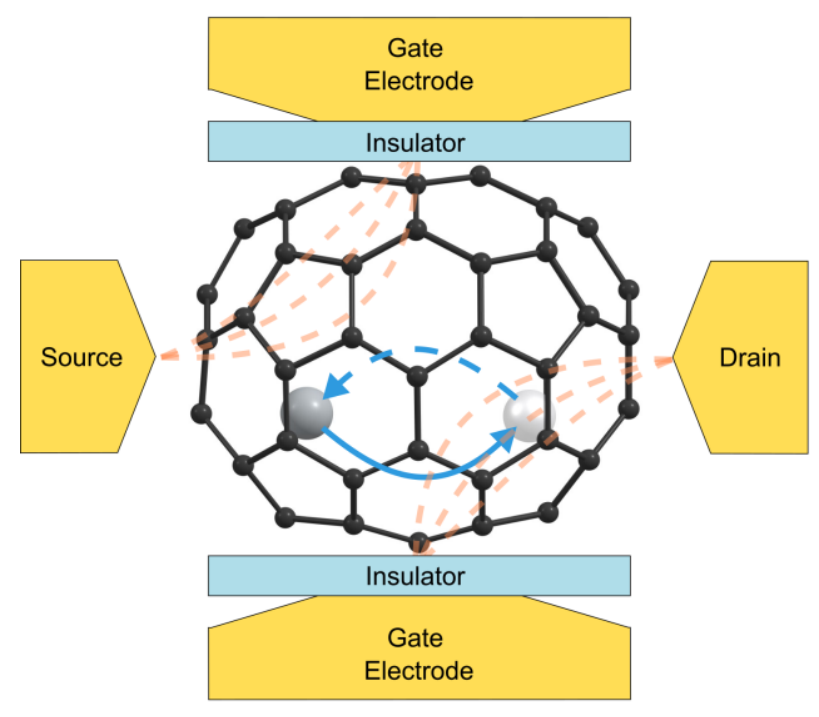

C
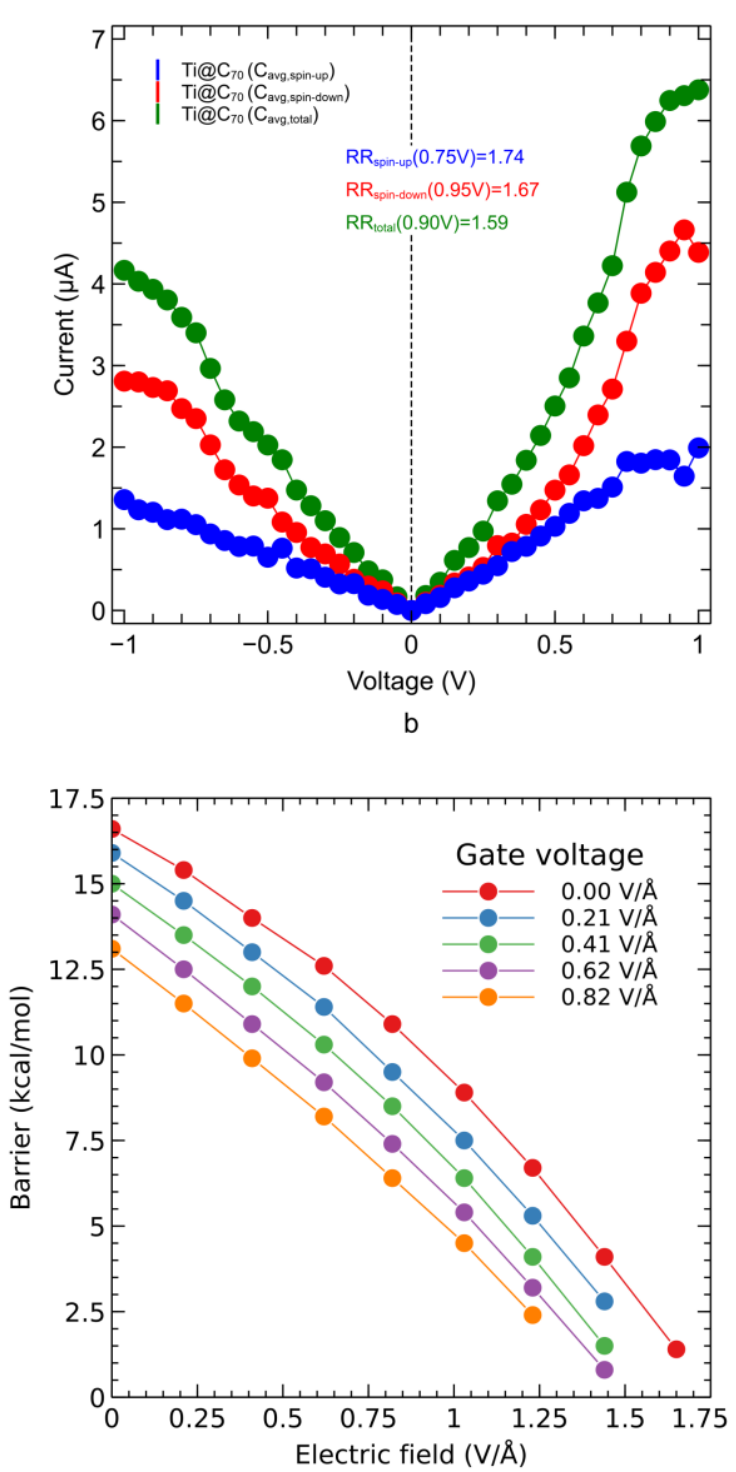

d

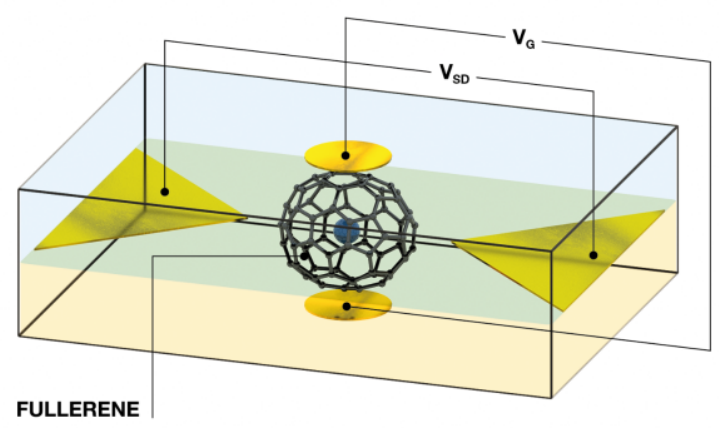

e

Fig. 1. The functions of $\mathrm{M} @ \mathrm{C}_{70}$ molecular spinristor. (a) A schematic representation of the fields applied during the switching process. (b) The variation of the switching barrier for relocation of titanium atom inside $\mathrm{Ti} @ \mathrm{C}_{70}$ versus the potential difference between source and drain electrodes under the effect of the gate potential, $V_{\mathrm{G}}$. (c) A schematic illustration of the spin-filtering process when $\mathrm{V}_{\mathrm{SD}}$ is applied. (d) The computed I/V characteristic of the Ti@ $\mathrm{C}_{70^{-}}$ $D_{5 h}(1)$. Averaged values for various connections of the system to electrodes. Note that the 
absolute values are reported here for the current for a more clear view of the spin asymmetry of the passing current, $\mathrm{ISD}_{\mathrm{SD}}$ (e) $3 \mathrm{D}$ representation of a spinristor. The position of the enclosed metal can be controlled through a quadrupolar field, applied via source, drain, and a pair of gate electrodes that are separated from the fullerene by two layers of an isolator material that fixes the elliptical fullerene in its position between source and drain electrodes.

To ensure a sufficiently large switching barrier in the proposed spinristor, we arrived at two model EMFs, Ti@ $\mathrm{C}_{70}-D_{5 h}(1)$ and $\mathrm{Zr} @ \mathrm{C}_{70}-D_{5 h}(1)$, that show field-free barriers for relocation of their metal of 16.6 and $23.0 \mathrm{kcal} . \mathrm{mol}^{-1}$, respectively. We utilized the fact that group 4 to 6 elements are known to form strong polar-covalent bonds to carbon atoms ${ }^{32,33}$ and that $\mathrm{C}_{70}-D_{5 h}(1)$ fullerene has an elliptical non-isotropic shape with distinct binding sites for the selected metal atoms. The proposed systems should combine reasonable switching barriers with spin-polarized electronic structures because of the enclosed metal, which brings in the spin-filtering function. However, in the case of $\mathrm{Zr} @ \mathrm{C}_{70}$, our computations predict that the external electric potential does not decrease the switching barrier to a level to be applicable in a real device. The $\mathrm{Zr} @ \mathrm{C}_{70}$ can still function as a spin filter. Therefore, we keep the discussion in the main text limited to the $\mathrm{Ti} @ \mathrm{C}_{70}-D_{5 h}(1)$ system while all data regarding the $\mathrm{Zr} @ \mathrm{C}_{70}$ are collected in $\mathbf{S I}$.

We note that the proposed molecules have not been synthesized so far and serve as models here. Nevertheless, a few analogous systems have been experimentally observed. In particular, Hf@ $\mathrm{C}_{84}$ has been isolated ${ }^{34}$ and Ti@ $\mathrm{C}_{\mathrm{n}}(\mathrm{n}=28,80,90)$, as well as Zr@C28, have been detected in mass-spectroscopy experiments. ${ }^{35,36}$

\section{Proof of concept; computations predict $\mathrm{Ti} @ \mathrm{C}_{70-}-D_{5 h}(1)$ to function as a molecular spinristor}

Density functional theory (DFT) computations using B97D3 functional predict the ground-state local minimum structure $\left(\mathrm{LM} 1 / \mathrm{LM}^{\prime}\right)$ of $\mathrm{Ti} @ \mathrm{C}_{70}$ to be a closed-shell singlet, with 
the lowest triplet state at just $3.5 \mathrm{kcal}_{\mathrm{mol}} \mathrm{m}^{-1}$ above it, Fig. 2a. The closed-shell singlet groundstate may seem to contradict the spin-filtering function. However, when connected to the electrodes the triplet is the ground state of the system. In LM1, the metal is connected to the interior of the fullerene cage in the electron-rich polar region. The position of the metal can switch between LM1 and LM1' as the metal atom passes through the cavity of the cage to the other side. To switch between the local minima, the $\mathrm{Ti} @ \mathrm{C}_{70}$ has to pass through the respective transition state (TS) that is computed to be $16.6 \mathrm{kcal}^{\mathrm{mol}}{ }^{-1}$ higher than LM1, Fig. 2a. An intermediate, labeled LM2, $10.5 \mathrm{kcal}_{\mathrm{mol}}{ }^{-1}$ above LM1 forms when the Ti is midway to the other side of the cage. The ground electronic state of the transition states between LM1/LM1' and LM2, $\left(\mathrm{TS} / \mathrm{TS}^{\prime}\right)$, is a triplet in the gas phase for $\mathrm{Ti} @ \mathrm{C}_{70}$. Therefore, an intersystem crossing occurs while the metal switches its position, Fig. 2b. For simplicity, we consider switching between LM1 and LM1'. It can be safely assumed that when the applied external electric field (EEF) is strong enough for LM1 to pass through TS, the system will not stop at LM2 as the barrier between LM2 and TS' is notably smaller than the one between LM1 and TS on the potential energy surface (PES) of the molecule, Fig. 2a.

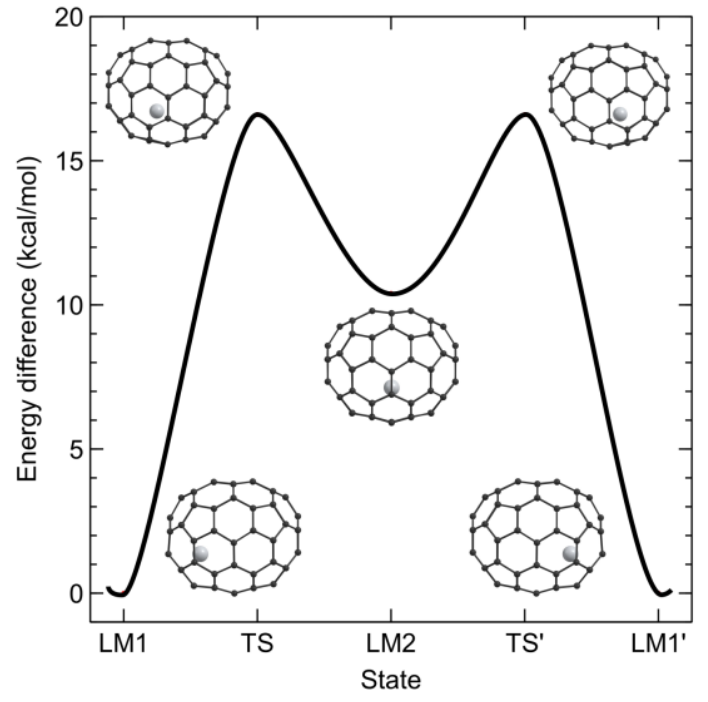

a

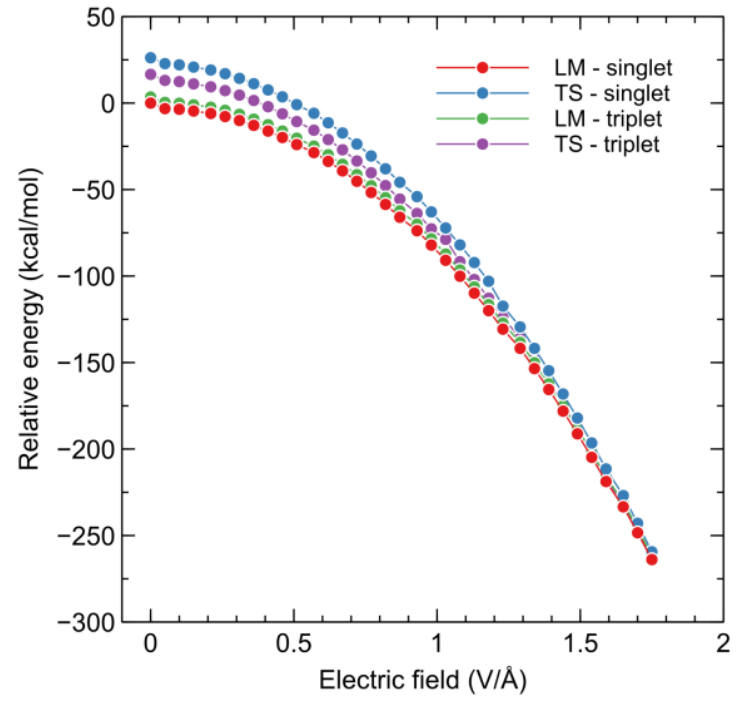

b

Fig. 2. (a) Schematic representation of the potential energy surface of Ti atom in $\mathrm{C}_{70}$. (b) The effect of an EEF on the relative energies of the transition state and local minima with respect to that of the local minimum in the absence of an EEF. 
Controlling the position of metals inside EMFs by an $\mathrm{EEF}^{15,37}$ can be realized via scanning tunneling microscopy ${ }^{25,26,29}$ or, as recently reported, in a circuit. ${ }^{16}$ In principle, an increasing EEF reduces and eventually removes the energy barrier for switching by destabilizing the local minimum structures, in which the dipole moment vector of the structures are parallel to and along with the polarity of the applied field, Fig. 1c. Simultaneously, the field stabilizes the local minima with a dipole moment vector against the direction of the externally applied field. Destabilization of one local minimum and stabilization of the other local minimum connected via a TS on a PES reduces the energy barriers between them as it is expected from the Hammond postulate. ${ }^{37}$ Additionally, the application of a quadrupolar field via the gate electrodes reduces the energy barrier further by manipulating the energies of TS/TS' or the intermediate LM2, directly; see Fig. 1d. We found that destabilizing LM2 is a more efficient way to achieve LM1' from LM1 than stabilizing TS/TS'.

We define the switching EEF as the field(s) (dipolar or quadrupolar) that is large enough to change the nature of a local minimum structure, e.g.LM1, on the PES, to a transition state or a non-stationary point so that the Ti relocates to another minimum position, e.g. LM1'. A strong and short pulse of an electric potential that is equal to the switching EEF switches the position of the Ti between LM1 and LM1' within the time scale of a single molecular vibration (within the domain of terahertz) by eradicating LM1 on the PES. ${ }^{15}$ The computed switching EEF for Ti@ $\mathrm{C}_{70}$ is ca $1.75 \mathrm{~V} . \AA^{-1}$ in the case of a uniform dipolar EEF in our model (see Methods), Fig. 1d. Using electrodes with sharp tips can intensify the electric field locally, and therefore the voltage needed to achieve the switching EEF can be reduced by an order of magnitude or even more. ${ }^{38}$ Using a quadrupolar electric field (Fig. 1c) with voltage at the gate electrodes further helps the system to pass through the intrinsic barrier by affecting the energies of LM2 and TS/TS', Fig. 1d. 
The EEF-control of the position of Ti enables writing/encoding information on the device. To read the encoded information from the device, one can measure transmission through the molecule using a low $\mathrm{V}_{\mathrm{SD}}$ applied between the source and drain electrodes, Fig. 1a. The DFT-coupled non-equilibrium Green's function (DFT-NEGF) computations predict that $\mathrm{Ti} @ \mathrm{C}_{70}$ behaves as a molecular rectifier and a spin-filter at the same time when $\mathrm{V}_{\mathrm{SD}}$ is applied, as illustrated in Fig. 1b. Different connection modes, presented in Fig. S2, lead to moderately different transmission properties but the resulting I-V curves share similar common features; see Fig. S5 for the I/V curves of each connection mode. The I/V characteristics reveal that Ti@ $\mathrm{C}_{70}$-based spinristor in its LM1/LM1' state/minima moderately filters the current passing through in the range of $\mathrm{V}_{\mathrm{SD}}= \pm 1 \mathrm{~V}$. On average, the maximum rectification for the spin-up, spin-down, and the total currents are predicted at different voltages $\left(R R_{\text {Max }}^{\text {total }}==1.59\right.$ at $\mathrm{V}_{\mathrm{SD}}=0.90 \mathrm{~V}, R R_{\operatorname{Max}}^{\text {spin-up }}=1.74$ at $\mathrm{V}_{\mathrm{SD}}=0.75 \mathrm{~V}$, and $R R_{\text {Max }}^{\text {spin-down }}=1.67$ at $\mathrm{V}_{\mathrm{SD}}=0.95$ V).

The origin of the spin-filtering and rectification properties can be traced to the electronic structure of the Ti@ $\mathrm{C}_{70}$ molecule, Fig. 3a, and b. Studying the transmission spectra at the voltages where the maximum rectification is observed suggests that the rectification for the spin-up electrons arises from the energy shift of the LUMO-type orbitals within the window of the applied bias voltage when a positive voltage is applied, Fig. 3a. Rectification of the spindown electrons however stems from the energy shifts of both the LUMO- and the HOMO-type MOs, Fig. 3b. Note that HOMO-type MOs have a more significant contribution to the transmission spectra within the bias voltage window, where the maximum rectification for spin-down electrons is observed. The spin- and orbital-resolved partial density of states, soPDOS, Fig. 3c, suggest that the contribution of the partially occupied $d$-orbitals of titanium, which are half-filled due to $\pi$-back donation from the carbon atoms to the enclosed titanium atom, is the origin of the spin-filtering properties, Table S1 and Fig. S3. Variation in the energy 
level of the MOs with the applied voltage changes the spin-filtering ability of the molecule from around $70 \%$ in the range of $\mathrm{V}_{\mathrm{SD}} \sim 0.05$ to $0.85 \mathrm{~V}$ to about $40 \%$ at $\mathrm{V}_{\mathrm{SD}} \sim 1.0 \mathrm{~V}$, Fig. 3d. The S-functionalized system is a better conductor for the spin-down electrons, it is a more efficient rectifier for the spin-up electrons.

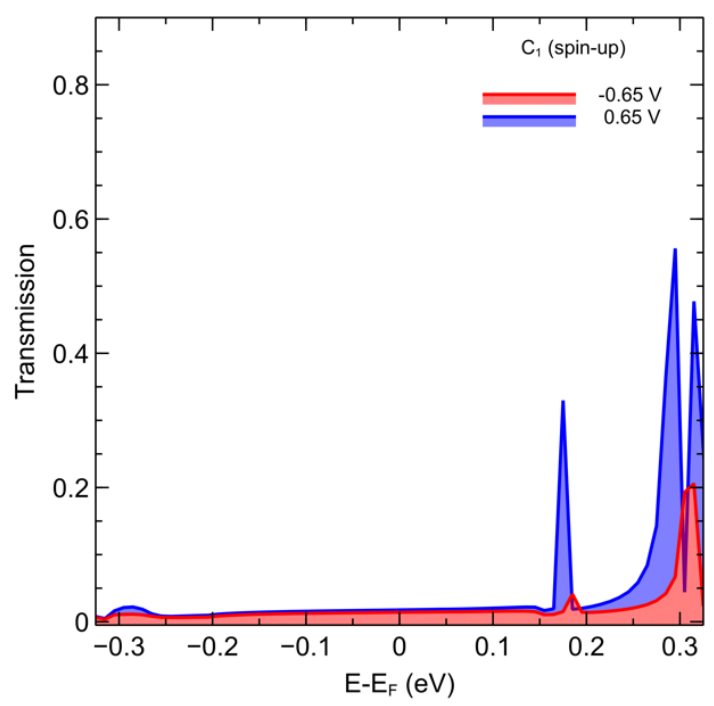

a

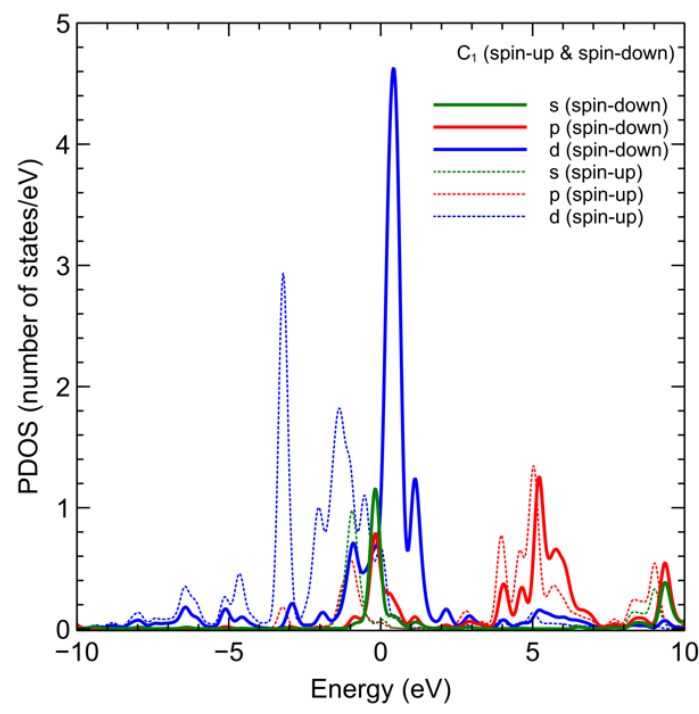

C

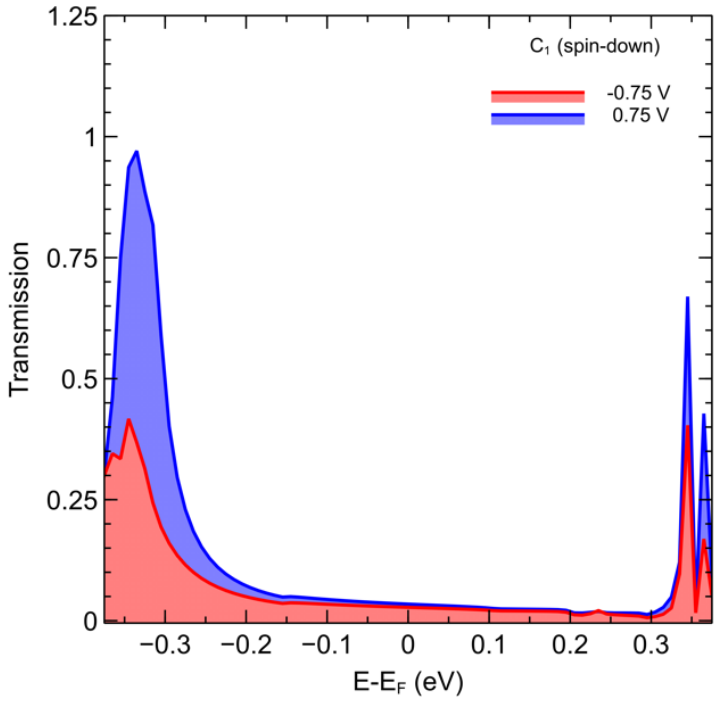

b

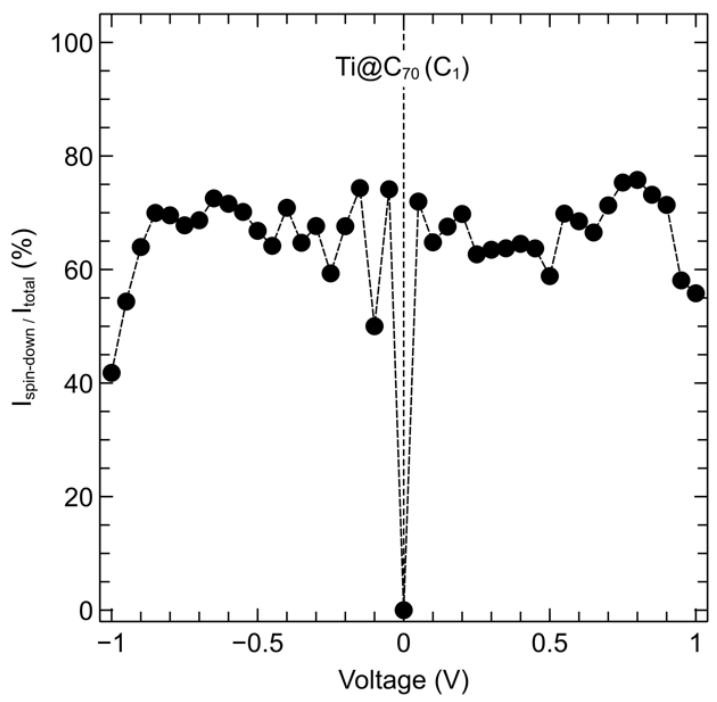

d

Fig. 3. Transmission spectra of $\mathrm{Ti} @ \mathrm{C}_{70}$ at the bias window at which the best rectification ratios are observed for spin-up (a) and spin-down (b) currents. (c) PDOS of the $\mathrm{Ti} @ \mathrm{C}_{70}$ in connection mode to electrodes C1, see Fig. S2). (d) The average percentage of spin filtering for all connection modes at various voltages. The highest spin filtering is observed at $\sim 0.8 \mathrm{~V}$ where nearly $80 \%$ of the passing current is spin-down. 


\section{Sulfur linkers strongly affect the spin-filtering function}

Sulfur functionalization is a routine approach to fabricate stable molecule-electrode connections via the formation of strong $\mathrm{Au}-\mathrm{S}$ bonds with the electrodes. Therefore, we further probed the effect of the sulfur functionalization of $\mathrm{Ti} @ \mathrm{C}_{70}$ on its transmission properties. Twenty-five possible isomers of dithiol derivate Ti@ $\mathrm{C}_{70}(\mathrm{SH})_{2}$ with sulfur atoms attached to the $\alpha$ carbons of the cage were optimized and the transmission properties of the two lowest energy isomers were computed; see Table $\mathbf{S 2}$ for the relative energies of the isomers. The conductivity of Ti@ $\mathrm{C}_{70} \mathrm{~S}_{2}$, in which the hydrogens of the thiol groups are removed to connect the sulfur covalently to the gold electrodes was evaluated. The calculated transmission through Ti@ $\mathrm{C}_{70} \mathrm{~S}_{2}$ is about a half of that of the unsubstituted Ti@ $\mathrm{C}_{70}$, Fig. 4a. However, the I/V curves of both the spin-up and the spin-down currents show remarkably new features. First, for low voltages, where $\left|\mathrm{V}_{\mathrm{SD}}\right|<0.5 \mathrm{~V}$, the positive voltage leads to a larger conductivity than the negative voltage. The maximum $\mathrm{RR}$ of $2.42,2.04$, and 2.15 is observed at $\mathrm{V}_{\mathrm{SD}}= \pm 0.3 \mathrm{~V}$ for the spin-up, spin-down, and total currents, respectively. At higher voltages, $\left|\mathrm{V}_{\mathrm{SD}}\right|>0.5 \mathrm{~V}$ the negative voltage region shows a more pronounced conductivity with rectification ratios of 9.32 $\left(\mathrm{V}_{\mathrm{SD}}=0.90 \mathrm{~V}\right), 3.92\left(\mathrm{~V}_{\mathrm{SD}}=0.80 \mathrm{~V}\right)$, and $4.63\left(\mathrm{~V}_{\mathrm{SD}}=0.90 \mathrm{~V}\right)$ for the spin-up, spin-down, and total currents, respectively, Fig. 4a. The reason for the change in the transmission properties of the system at different voltages is switching among different conductance channels. At low voltages, a SOMO-type MO on the Fermi level is the main conductance channel of the molecule as it is evident from the transmission spectra at $\mathrm{V}_{\mathrm{SD}}= \pm 0.3 \mathrm{~V}$, Figs. 5ab. As the bias voltage increases, the SOMO-type orbital shifts away from the Fermi level, and LUMO-type MOs start to contribute to the conductivity of spin-up electrons while HOMO-type ones give the pass to the spin-down electrons, Figs. 5cd. 

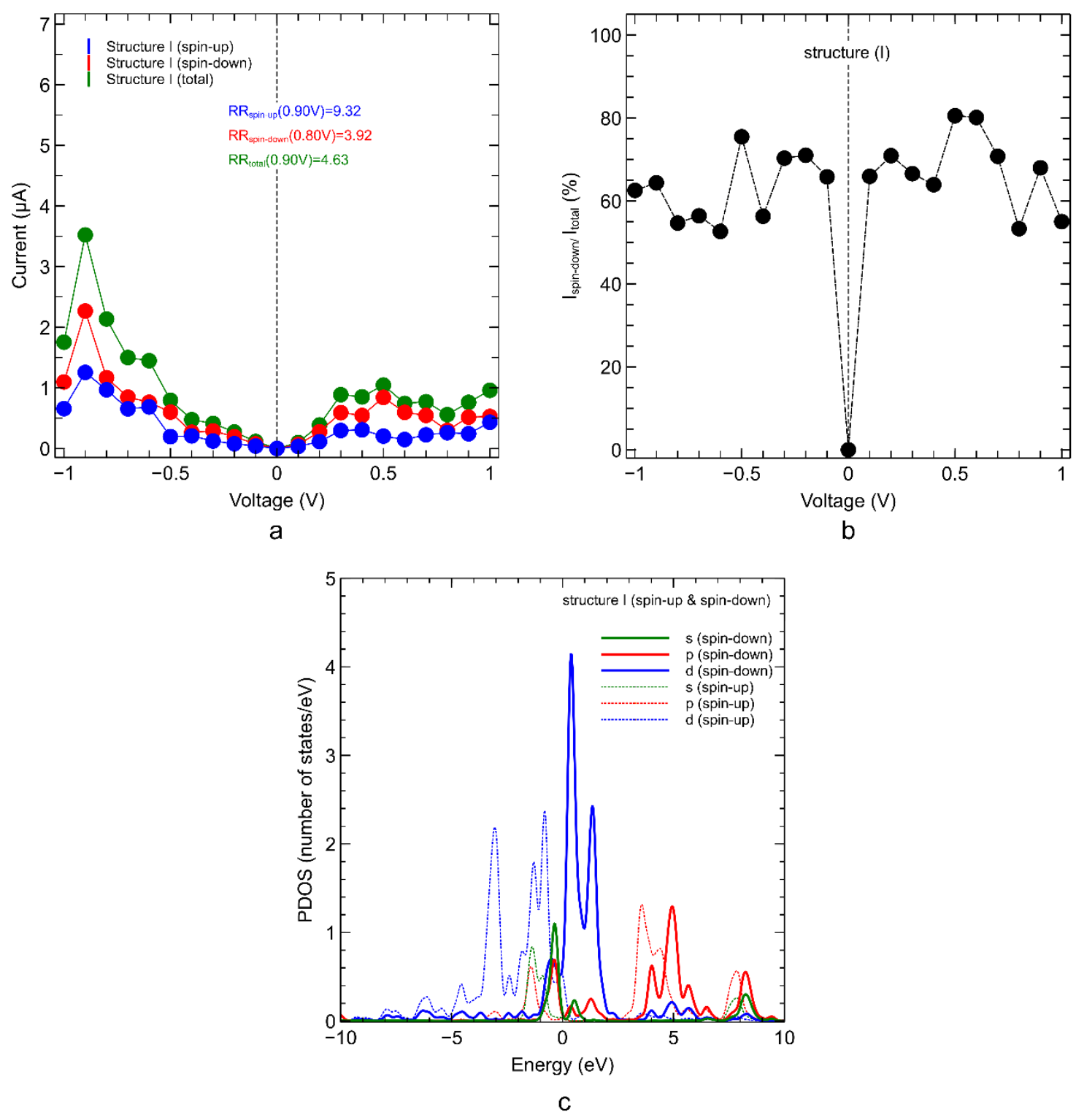

Fig. 4. (a) The computed I/V characteristic of the Ti@ $\mathrm{C}_{70} \mathrm{~S}_{2}$. (b) Percentage of spin filtering for the lowest energy isomer of $\mathrm{Ti} @ \mathrm{C}_{70} \mathrm{~S}_{2}$.

S-functionalization does not change the spin-filtering performance of the $\mathrm{Ti} @ \mathrm{C}_{70}$ system, Fig. 4b. Finally, the spin- and orbital-resolved partial density of states suggest that in the $\mathrm{Ti} @ \mathrm{C}_{70} \mathrm{~S}_{2}$ system the spin conductivity is the result of the contribution of the $d$-orbitals of the titanium atom, Fig. $\mathbf{4 c}$, akin to the parent compound. 


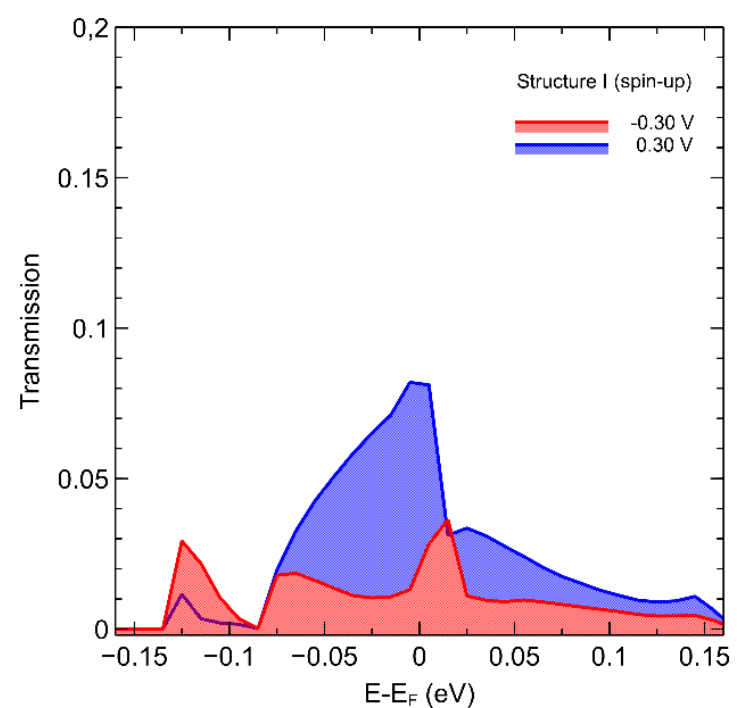

a

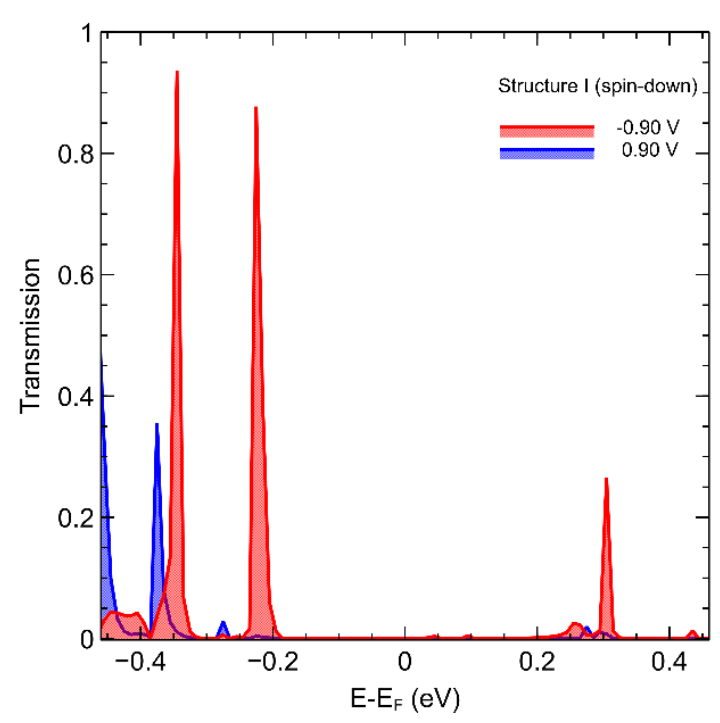

C

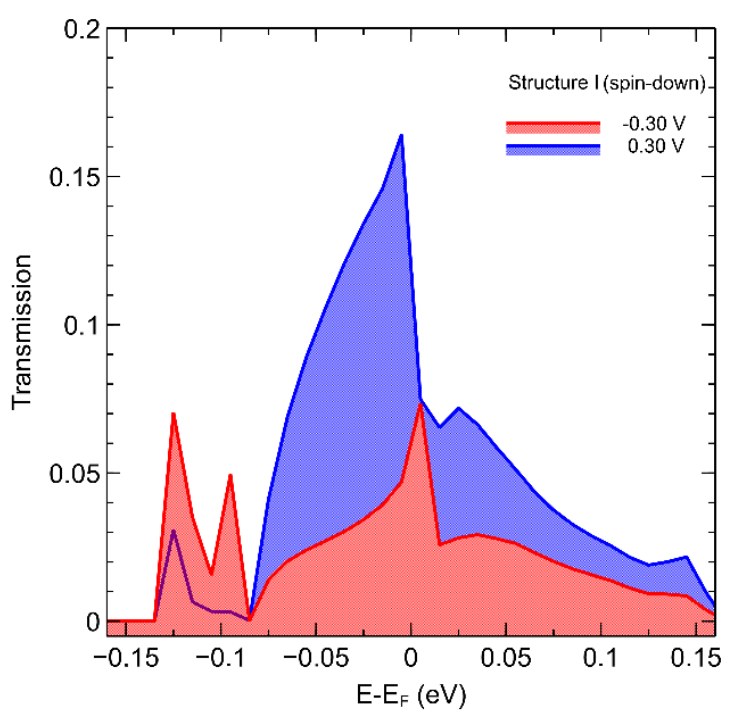

b

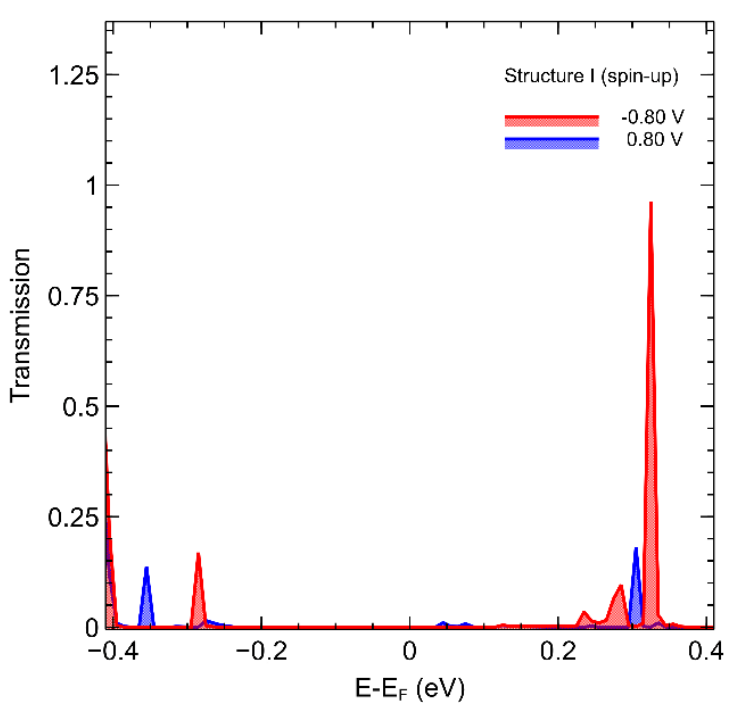

d

Fig. 5. Transmission spectra of $\mathrm{Ti} @ \mathrm{C}_{70} \mathrm{~S}_{2}$ at the bias windows at which the best rectification ratios are observed for spin-up (a,c) and spin-down (b,d) currents. (e) PDOS of the lowest energy isomer of $\mathrm{Ti} @ \mathrm{C}_{70} \mathrm{~S}_{2}$.

\section{Conclusions and Prospects: Looking for the spinristors on a macroscopic}

\section{scale.}

In summary, employing in silico calculations we proposed, designed, and provided a proof-ofconcept of the function of a molecular spinristor, which is a circuit component reported neither in the realm of molecular nor macroscopic electronics, to the best of our knowledge. The spinristor behaves like a combination of a spin filter and a rectifier at low voltages. At higher 
voltages, the polarity of the rectifier switches akin to a memristor by the relocation of the encapsulated metal ion. Our design utilizes $\mathrm{M} @ \mathrm{C}_{70}$ molecules connected to four electrodes, source, drain, and a pair of gate electrodes to improve the switching function. The spin-filtering originates from the open-shell electronic structure of the system with half-filled $d$-orbitals when attached to a pair of electrodes. The rectification of the molecule is the result of the metal encapsulation that polarizes the MOs of the fullerene, like a molecular memristor. ${ }^{15}$ Finally, the switching stems from the conversion between the two most stable configurations of the endohedral metallofullerene, where the metal ion is near the polar region of the cage. According to the NEGF computations, sulfur-functionalization improves rectification properties of the molecule significantly, while spin-filtering function remains similar.

As mentioned above, the concept of spinristor, unlike other circuit components, is for the first time theoretically predicted for a single-molecule device. However, this does not rule out the possibility of finding spinristive materials on a macroscopic scale. Contemporary experimental memristors are often composed of halides or chalcogenides of metals and semimetals. ${ }^{39}$ Possibly, magnetic halides or chalcogenides of magnetic metals may open a way to the fabrication of macroscopic scale spinristors. Such materials may combine the ion-mobility of the memristive materials with magnetic properties of their bulk to represent the spinristive behavior. Presently, we are not aware of any studies in this direction.

\section{Methods}

Molecular structures were optimized using Gaussian 16 suite of programs ${ }^{40}$ at the B97D3 $3^{41,42} /$ def2-SVP ${ }^{43,44}$ computational level. ${ }^{45,46}$ The stationary points on the potential energy surface of the molecules were verified as minima and transition states by evaluating the harmonic vibrational frequencies. The most abundant isolated-pentagon rule (IPR) $\mathrm{C}_{70}-D_{5 h}: 1$ cage was used in studies. The effect of the oriented external electric field on the energy and structures of the species was studied by applying uniform EF along the main $D_{5}$ axis of symmetry of $\mathrm{C}_{70}$. A second $\mathrm{EF}$ perpendicular to the 
initial EF, as it is implemented under the Field option of the Gaussian 16 software, was applied to model a gate voltage effect on the energy and geometry. Energy profiles corresponding to the translation of titanium ion inside $\mathrm{C}_{70}$ were studied both by minimum/transition state computations and also by relaxed PES scan approaches to catch all possible minima, Fig. S9.

To perform non-equilibrium Green's function (NEGF) computations we dissected our systems into three regions, that consist of a scattering region and two semi-infinite electrodes. No direct interaction between the electrodes was considered. The $\mathrm{Au}(001)$ surface of a bulk gold structure was selected to utilize the electrodes. Each electrode consists of 5 and 4 gold layers in sequence, and a single $\mathrm{Au}$ atom as the tip of the electrode. The optimized system was embedded between two gold atoms at the tips of the electrodes via the $\alpha$-carbon atoms of the fullerene. Five possible connection modes were considered, see Fig. S2. We fixed the distance between the tip and the $\alpha-\mathrm{C}$ to $2.33 \AA$ that is the optimized bond length of $\alpha-\mathrm{C}-\mathrm{Au}$ at $\mathrm{PBE}^{47} / \mathrm{DZP}^{48}$ computational level as described elsewhere. ${ }^{15}$

DFT-NEGF computations using generalized gradient approximation (GGA) functional developed by Perdew, Burke, and Ernzerhof $(\mathrm{PBE})^{47}$ as implemented in the TranSiesta package ${ }^{48}$ combined with double- $\zeta$ polarized basis set as implemented in Siesta suite of programs were used for all electron transport computations. ${ }^{49}$ The energy cut-off was set to 300 Ry for the real space grid. $\Gamma$ points for sampling were used for the first Brillouin zone in the molecular region and $1 \times 1 \times 100$ Monkhorst-Pack k-point grid for the nanowire electrodes. ${ }^{50,51}$ All the transport properties were carried out for the applied voltage in the range of $\pm 1 \mathrm{~V}$. The electron distribution through the main eigenchannels, that is the expectation value of the eigenchannels greater than $10^{-5}$, were analyzed using Inelastica code in which both sides, left to right and vice versa are considered in addition to the imaginary part..$^{52}$

\section{Acknowledgments}

Computational resources were provided by CESNET (LM2015042), the CERIT Scientific Clouds (LM2015085). This work was supported by Czech Science Foundation Grant 21-17806S to MS. CFN thanks to National Science Centre, Poland 2020/39/B/ST4/02022 for funding this 
work. For Open Access, the authors have applied a CC-BY public copyright license to any Author Accepted Manuscript (AAM) version arising from this submission. The authors thank Tomáš Belloň for providing the TOC graphics.

\section{Data availability}

Electronic properties of $\mathrm{Ti}_{\mathrm{C}} \mathrm{C}_{70}$ (Table S1); switching properties of the $\mathrm{Zr} @ \mathrm{C}_{70}$ system (Fig. S1); numbering of carbon atoms used for transmission properties (Fig. S2); spin density of $\mathrm{Ti}_{0} \mathrm{C}_{70}(\mathbf{F i g}$. S3); partial density of states (Fig. S4); I/V characteristics (Fig. S5); relative energies of $\operatorname{Ti}_{0} \mathrm{C}_{70}(\mathrm{SH})_{2}$ isomers (Table S2); spin filtering properties of $\mathrm{Ti} @ \mathrm{C}_{70} \mathrm{~S}_{2}($ Fig. S6); spin filtering properties in various connection modes (Fig. S7); transmission spectra for all connection modes (Fig. S8); relaxed scan of titanium atom translation through the cage (Fig. S9). The Cartesian coordinates of LM1, LM2, TS, and Ti @ $\mathrm{C}_{70}(\mathrm{SH})_{2}$ isomers as well as the coordinates of systems for Transiesta computations are presented.

\section{Author Contributions}

EFB and AJ contributed equally to this work. EFB, ZB, and LT performed NEGF calculations. AJ and LT performed electric field calculations. EFB and CFN provided the initial idea. CFN and MS have coordinated the project and participated in writing.

\section{Competing interests}

The authors declare no competing interest

\section{References}

1. Joachim, C., Gimzewski, J. K. \& Aviram, A. Electronics using hybrid-molecular and monomolecular devices. Nature 408, 541-548 (2000).

2. Mark Ratner. A brief history of molecular electronics. Nat. Nanotechnol. 8, 378-381 (2013).

3. Xiang, D., Wang, X., Jia, C., Lee, T. \& Guo, X. Molecular-Scale Electronics: From Concept to Function. Chem. Rev. 116, 4318-4440 (2016). 
4. Vilan, A., Aswal, D. \& Cahen, D. Large-Area, Ensemble Molecular Electronics: Motivation and Challenges. Chem. Rev. 117, 4248-4286 (2017).

5. Su, T. A., Neupane, M., Steigerwald, M. L., Venkataraman, L. \& Nuckolls, C. Chemical principles of single-molecule electronics. Nat. Rev. Mater. 1, 16002 (2016).

6. Chen, H. \& Fraser Stoddart, J. From molecular to supramolecular electronics. Nat. Rev. Mater. 6, 804-828 (2021).

7. Feynman, R. P. There is plenty of room at the bottom. vol. 23 22-36 (1960).

8. Aviram, A. \& Ratner, M. A. Molecular rectifiers. Chem. Phys. Lett. 29, 277-283 (1974).

9. Bumm, L. A. et al. Are Single Molecular Wires Conducting? Science 271, 1705-1707 (1996).

10. Díez-Pérez, I. et al. Rectification and stability of a single molecular diode with controlled orientation. Nat. Chem. 1, 635-641 (2009).

11. Andrews, D. Q., Solomon, G. C., Van Duyne, R. P. \& Ratner, M. A. Single Molecule Electronics: Increasing Dynamic Range and Switching Speed Using Cross-Conjugated Species. J. Am. Chem. Soc. 130, 17309-17319 (2008).

12. Jia, C. et al. Covalently bonded single-molecule junctions with stable and reversible photoswitched conductivity. Science 352, 1443-1445 (2016).

13. Tans, S. J., Verschueren, A. R. M. \& Dekker, C. Room-temperature transistor based on a single carbon nanotube. Nature 393, 49-52 (1998).

14. Miyamachi, T. et al. Robust spin crossover and memristance across a single molecule. Nat. Commun. 3, 938 (2012).

15. Jaroš, A., Bonab, E. F., Straka, M. \& Foroutan-Nejad, C. Fullerene-Based Switching Molecular Diodes Controlled by Oriented External Electric Fields. J. Am. Chem. Soc. 141, 19644-19654 (2019).

16. Zhang, K. et al. A Gd@C 82 single-molecule electret. Nat. Nanotechnol. 1-6 (2020) doi:10.1038/s41565-020-00778-z.

17. Li, H. B., Tebikachew, B. E., Wiberg, C., Moth-Poulsen, K. \& Hihath, J. A Memristive Element Based on an Electrically Controlled Single-Molecule Reaction. Angew. Chem. Int. Ed. 59, 11641$11646(2020)$. 
18. Paul, N. D., Rana, U., Goswami, S., Mondal, T. K. \& Goswami, S. Azo Anion Radical Complex of Rhodium as a Molecular Memory Switching Device: Isolation, Characterization, and Evaluation of Current-Voltage Characteristics. J. Am. Chem. Soc. 134, 6520-6523 (2012).

19. Goswami, S. et al. Robust resistive memory devices using solution-processable metalcoordinated azo aromatics. Nat. Mater. 16, 1216-1224 (2017).

20. Bergren, A. J. et al. Musical molecules: the molecular junction as an active component in audio distortion circuits. J. Phys. Condens. Matter 28, 094011 (2016).

21. Chua, L. O. \& Kang, S. M. Memristive devices and systems. Proc. IEEE 64, 209-223 (1976).

22. Strukov, D. B., Snider, G. S., Stewart, D. R. \& Williams, R. S. The missing memristor found. Nature 453, 80-83 (2008).

23. Abraham, I. The case for rejecting the memristor as a fundamental circuit element. Sci. Rep. 8 , $10972(2018)$.

24. Jia, C. et al. Conductance Switching and Mechanisms in Single-Molecule Junctions. Angew. Chem. Int. Ed. 52, 8666-8670 (2013).

25. Huang, T. et al. A Molecular Switch Based on Current-Driven Rotation of an Encapsulated Cluster within a Fullerene Cage. Nano Lett. 11, 5327-5332 (2011).

26. Yasutake, Y., Shi, Z., Okazaki, T., Shinohara, H. \& Majima, Y. Single Molecular Orientation Switching of an Endohedral Metallofullerene. Nano Lett. 5, 1057-1060 (2005).

27. Roukala, J., Straka, M., Taubert, S., Vaara, J. \& Lantto, P. Ratcheting rotation or speedy spinning: EPR and dynamics of Sc3C2@C80. Chem. Commun. 53, 8992-8995 (2017).

28. Popov, A. A., Yang, S. \& Dunsch, L. Endohedral Fullerenes. Chem. Rev. 113, 5989-6113 (2013).

29. Chandler, H. J., Stefanou, M., Campbell, E. E. B. \& Schaub, R. Li@C 60 as a multi-state molecular switch. Nat. Commun. 10, 2283 (2019).

30. Williams, R. S. Summary of the Faraday Discussion on New memory paradigms: memristive phenomena and neuromorphic applications. Faraday Discuss. 213, 579-587 (2019).

31. Darwish, N., Foroutan-Nejad, C., Domulevicz, L., Hihath, J. \& Díez-Pérez, I. CHAPTER 5:Principles of Molecular Devices Operated by Electric Fields. in Effects of Electric Fields on 
Structure and Reactivity (eds. Shaik, S. \& Stuyver, T.) 147-194 (2021).

doi:10.1039/9781839163043-00147.

32. Oyama, S. T. Introduction to the chemistry of transition metal carbides and nitrides. in The Chemistry of Transition Metal Carbides and Nitrides (ed. Oyama, S. T.) 1-27 (Springer Netherlands, 1996). doi:10.1007/978-94-009-1565-7_1.

33. Kiani, S., Yang, J.-M. \& Kodambaka, S. Nanomechanics of Refractory Transition-Metal Carbides: A Path to Discovering Plasticity in Hard Ceramics. J. Am. Ceram. Soc. 98, 2313-2323 (2015).

34. Akiyama, K. et al. New fullerenes of a group IV element: Hf metallofullerenes. Chem. Phys. Lett. 317, 490-496 (2000).

35. Cao, B. et al. EELS and 13C NMR Characterization of Pure Ti2@C80 Metallofullerene. J. Am. Chem. Soc. 123, 9679-9680 (2001).

36. Dunk, P. W. et al. The Smallest Stable Fullerene, M@C28 (M = Ti, Zr, U): Stabilization and Growth from Carbon Vapor. J. Am. Chem. Soc. 134, 9380-9389 (2012).

37. Foroutan-Nejad, C., Andrushchenko, V. \& Straka, M. Dipolar molecules inside C70: an electric field-driven room-temperature single-molecule switch. Phys. Chem. Chem. Phys. 18, 3267332677 (2016).

38. Aragonès, A. C. et al. Electrostatic catalysis of a Diels-Alder reaction. Nature 531, 88-91 (2016).

39. Yang, J. J., Strukov, D. B. \& Stewart, D. R. Memristive devices for computing. Nat. Nanotechnol. 8, 13-24 (2013).

40. Frisch, M. J. et al. Gaussian 16. (Gaussian, Inc., 2016).

41. Grimme, S., Ehrlich, S. \& Goerigk, L. Effect of the damping function in dispersion corrected density functional theory. J. Comput. Chem. 32, 1456-1465 (2011).

42. Grimme, S. Semiempirical GGA-type density functional constructed with a long-range dispersion correction. J. Comput. Chem. 27, 1787-1799 (2006).

43. Weigend, F. \& Ahlrichs, R. Balanced basis sets of split valence, triple zeta valence and quadruple zeta valence quality for H to Rn: Design and assessment of accuracy. Phys. Chem. Chem. Phys. 7, 3297-3305 (2005). 
44. Weigend, F. Accurate Coulomb-fitting basis sets for H to Rn. Phys. Chem. Chem. Phys. 8, 10571065 (2006).

45. Foroutan-Nejad, C., Straka, M., Fernández, I. \& Frenking, G. Buckyball Difluoride F2-@C60+-A Single-Molecule Crystal. Angew. Chem. Int. Ed. 57, 13931-13934 (2018).

46. Jaroš, A. et al. How Does a Container Affect Acidity of its Content: Charge-Depletion Bonding Inside Fullerenes. Chem. - Eur. J. 24, 4245-4249 (2018).

47. Perdew, J. P., Burke, K. \& Ernzerhof, M. Generalized Gradient Approximation Made Simple [Phys. Rev. Lett. 77, 3865 (1996)]. Phys. Rev. Lett. 78, 1396-1396 (1997).

48. Brandbyge, M., Mozos, J.-L., Ordejón, P., Taylor, J. \& Stokbro, K. Density-functional method for nonequilibrium electron transport. Phys. Rev. B 65, 165401 (2002).

49. Junquera, J., Paz, Ó., Sánchez-Portal, D. \& Artacho, E. Numerical atomic orbitals for linearscaling calculations. Phys. Rev. B 64, 235111 (2001).

50. Monkhorst, H. J. \& Pack, J. D. Special points for Brillouin-zone integrations. Phys. Rev. B 13, 5188-5192 (1976).

51. Pack, J. D. \& Monkhorst, H. J. 'Special points for Brillouin-zone integrations'-a reply. Phys. Rev. B 16, 1748-1749 (1977).

52. Paulsson, M. \& Brandbyge, M. Transmission eigenchannels from nonequilibrium Green's functions. Phys. Rev. B 76, 115117 (2007). 


\section{Table of contents graphics}

A metallofullerene can act as a molecular electronic component that enables switching between two distinctly different spin-filtering functions.

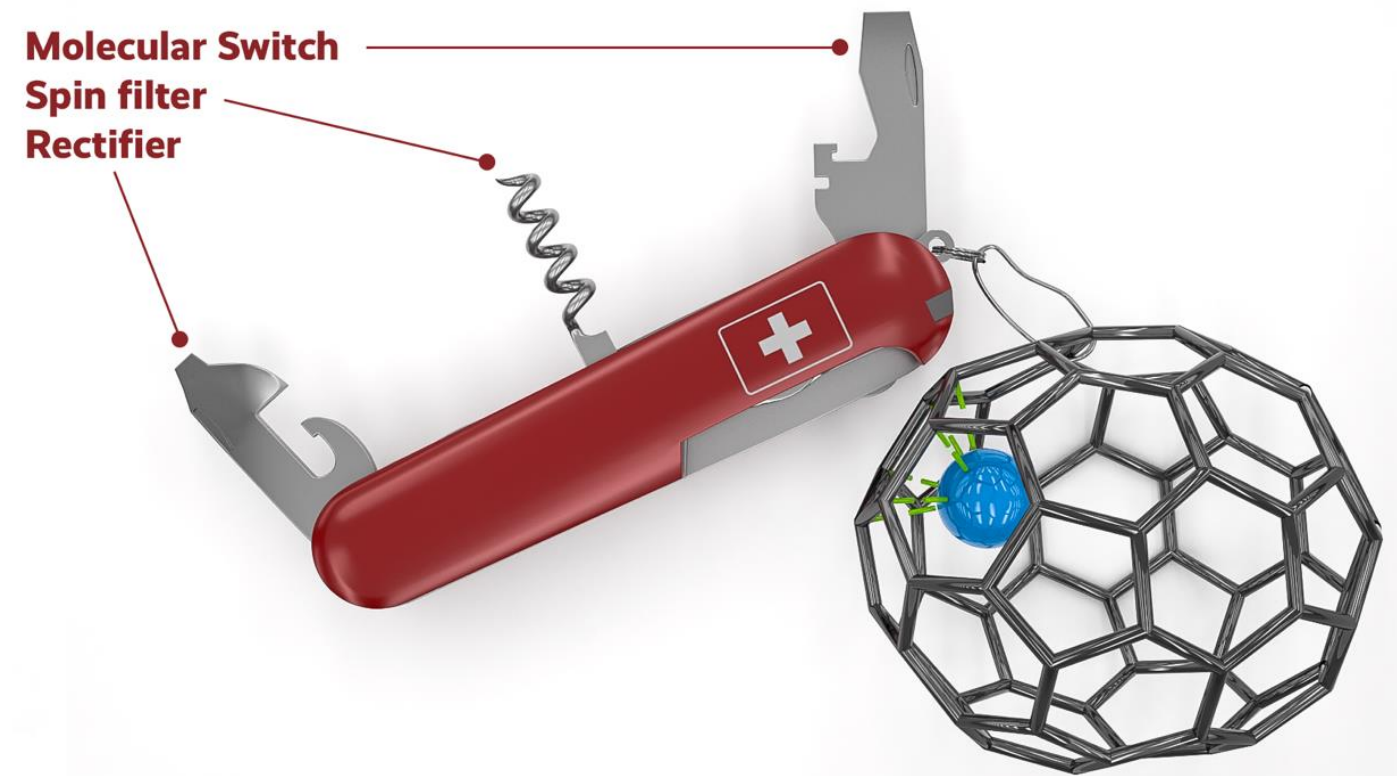

